Proceedings

\title{
Electrospun Polydioxanone Templates Loaded with Chloroquine Modulate Template-Induced NET Release and the Inflammatory Response ${ }^{\dagger}$
}

\author{
Allison E. Fetz, Shannon E. Wallace and Gary L. Bowlin *
}

Citation: Fetz, A.E.; Wallace, S.E.; Bowlin, G.L. Electrospun Polydioxanone Templates Loaded with Chloroquine Modulate Template-Induced NET Release and the Inflammatory Response. 2021, 78, 10. https://doi.org/10.3390/ IECP2020-08706

Published: 1 December 2020

Publisher's Note: MDPI stays neutral with regard to jurisdictional claims in published maps and institutional affiliations.

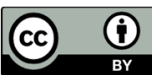

Copyright: $(C) 2021$ by the authors. Licensee MDPI, Basel, Switzerland. This article is an open access article distributed under the terms and conditions of the Creative Commons Attribution (CC BY) license (http://creativecommons.org/licenses/by/4.0/).

\author{
Department of Biomedical Engineering, University of Memphis, Memphis, TN 38152, USA; \\ aefetz@memphis.edu (A.E.F.); swllace8@memphis.edu (S.E.W.) \\ * Correspondence: glbowlin@memphis.edu \\ † Presented at the 1st International Electronic Conference on Pharmaceutics, 1-15 December 2020; Available \\ online: https://iecp2020.sciforum.net/.
}

\begin{abstract}
Electrospun biomaterials for in situ tissue regeneration are advantageous as drug delivery systems because they can be used to regulate the acute inflammatory response to establish a regenerative microenvironment. Neutrophils are the first inflammatory cells recruited to an electrospun biomaterial, and their release of neutrophil extracellular traps (NETs) and secretion of inflammatory and regenerative signals modulate the microenvironment around the biomaterial. In this work, chloroquine diphosphate, an anti-inflammatory drug shown to decrease NET release, was electrospun into polydioxanone (PDO) fibers for local delivery and immunomodulation of biomaterialinteracting neutrophils. Electrospinning was optimized so that the eluted concentration of chloroquine reached a previously reported, therapeutic concentration of $10 \mu \mathrm{M}$ within one hour. Subsequently, acute neutrophil-template interactions were evaluated in vitro with freshly isolated human peripheral blood neutrophils for 3 and $6 \mathrm{~h}$. NET release was quantified through detection of NETderived myeloperoxidase with an ELISA, and the secretion of inflammatory and regenerative factors was quantified with an immunomagnetic multiplex assay. The results indicate that the chloroquine-eluting templates significantly reduced NET release within the first $3 \mathrm{~h}$, but had no effect at $6 \mathrm{~h}$, suggesting a therapeutic window for modulating acute NET release or temporal changes in cell viability. Additionally, the elution of chloroquine increased the secretion of regenerative factors HGF, VEGF-A, and IL-22 while suppressing inflammatory signals MMP-9 and IL-8 at 3 and $6 \mathrm{~h}$, indicating a shift towards a pro-healing neutrophil phenotype. Together, these data suggest that chloroquine-eluting PDO biomaterials may modulate the acute neutrophil response from inflammatory to pro-healing, which may significantly enhance in situ tissue regeneration. Future work includes in vivo studies to evaluate long-term effects on tissue integration and regeneration in a physiological environment. Ultimately, these electrospun biomaterials may function as immunomodulatory drug delivery systems that regulate the neutrophil response and enhance the potential for in situ tissue regeneration.
\end{abstract}

Keywords: electrospinning; drug delivery; NETosis

Institutional Review Board Statement: Ethical review and approval were waived for this study since purchased or donated samples are not traceable back to the donor and does not qualify as human subjects research as determined by the University of Memphis Institutional Review Board on 22 November 2016. 